\title{
Infantile Fibrosarcoma of Tongue: A Rare Tumor
}

\author{
Fazal I Wahid, * Bakht Zada, Gul Rafique
}

\begin{abstract}
Infantile fibrosarcoma is very rare tumor and seldom occurs in oral cavity. Overall good prognosis is reported in more than $80 \%$ cases. We present a 5-month old female patient with swelling of the tongue for four months. This was excised completely. Histopathology and immunohistochemistry revealed it as infantile fibrosarcoma of grade II.
\end{abstract}

Key words: Fibrosarcoma; Infantile fibrosarcoma; Soft tissue tumor

\section{CASE REPORT}

A 5-month old female infant presented with progressive swelling of the tongue since one month of age. There was no associated difficulty in swallowing or breathing. On examination, there was a firm, diffuse swelling about $3 \mathrm{~cm} \times 4 \mathrm{~cm}$ in dimension, involving left side of the tongue and extending to midline. Mucosa over the mass was intact and margins were ill-defined (Fig. 1).

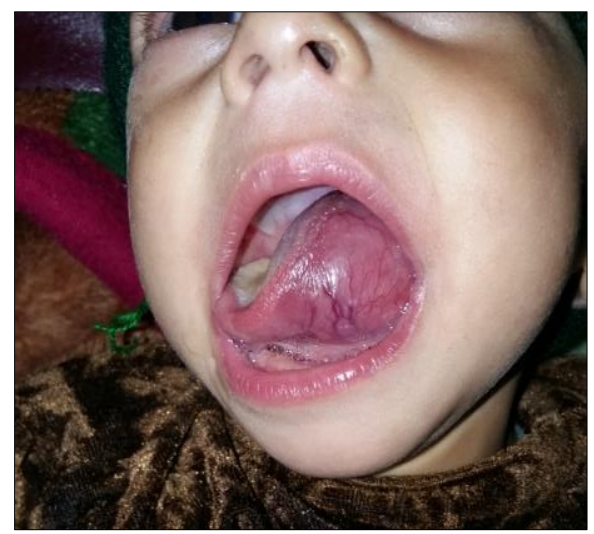

Figure 1: Five month old baby with swelling of the tongue.

There was no associated cervical lymphadenopathy. Ultrasonography revealed heterogeneous mass in the tongue sparing the floor of mouth. Fine needle aspiration was inconclusive. At operation adrenaline mixed local anesthetic was injected at the incision site to minimize the bleeding. Linear incision was made on the left lateral border of the tongue. The mass was excised and wound stitched with polyglycolic 3/0. Patient was put on injectable antibiotics for 48 hours with mouth cleansing antiseptic oral spray. Specimen was sent for histopathology which was reported as infantile fibrosarcoma of grade II. The margins of the excision were tumor free. Immunohistochemical staining showed positive staining for SMA and CD34 which also supported the diagnosis. Patient was reviewed postoperatively and referred to oncologist for chemo-therapy (Fig. 2). At three months follow up patient was disease free after receiving chemotherapy.

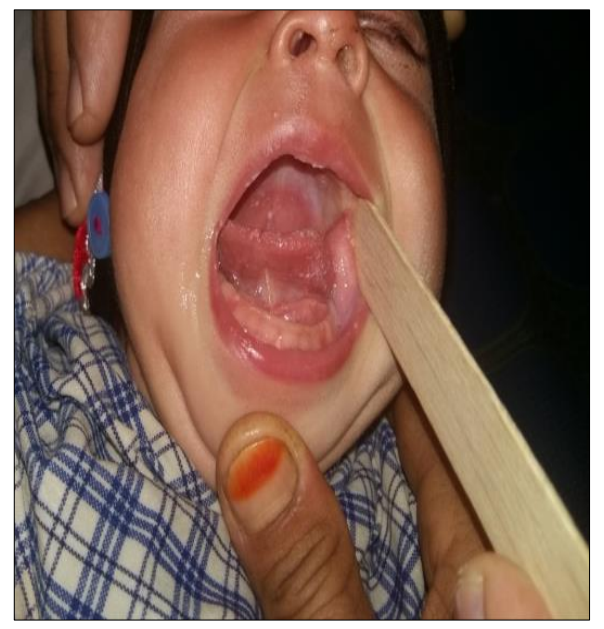

Figure 2: Post-operative picture after excision of the mass.

\section{DISCUSSION}

Fibrosarcomas are malignant tumors predominantly originating from soft tissues.[1] Fibrosarcomas are commonly found in the extremities, trunk, and head and neck regions in descending order of frequency. Among all the fibrosarcomas, only $0.05 \%$ occur in head and 
neck region, of which $23 \%$ occur in oral cavity.[2] Rarely it arises from the tongue.[2-5] There are two types of fibrosarcomas, infantile or congenital (IF) and adult form. The presentation of congenital fibrosarcoma is usually in early age as in our case. Clinical features depend upon the size, site and grade of the tumor. However, it presents as slow growing painless mass.[6] Feeding and breathing difficulties are the main concerns in case of intraoral mass lesions. In our patient the mass was also increasing slowly in size but it was not causing any feeding or breathing difficulties. The exact cause of IF remains obscure, however genetic malformation and radiation may contribute.[3]

CT scan or MRI may point a solid mass but the exact diagnosis can only be made on histopathology powered by immunohistochemistry as we diagnosed in our case.[2,6,7] Infantile fibrosarcoma can be graded into low, intermediate and high grade depending on severity of cellularity, nuclear pleomorphism and mitosis.[3] Infantile fibrosarcoma must be kept in the differentia diagnosis of other soft tissues tumors like rhabdomyosarcoma and Infantile myofibromatosis and its variants. $[4,8]$ Our case was of grade II on histopathology. The management of IF is a multidisciplinary which includes surgery, chemotherapy and radiotherapy with rehabilitation if indicated.[4] Surgery is the mainstay of treatment for IF. In our case the mass was excised apparently completely and then patient was referred to oncologist. The success of treatment of IF depends on age of the patient, extent and site, grade and stage of the disease, response to the treatment and associated side effects of the medications. Overall good prognosis of IF is more than $80 \%$, if treated in time.[8] Our patient is doing well till date. In conclusion, although infantile fibrosarcoma is a rare tumour, it must be kept in differential diagnosis of soft tissue tumors presenting in infancy. Surgery is the mainstay of treatment for infantile fibrosarcoma that can be complemented with chemo-radiotherapy depending upon its stage.

\section{REFERENCES}

1. Orbach D, Rey A, Cecchetto G, Oberlin O, Casanova M, Thebaud $E$, et al. Infantile fibrosarcoma: management based on the European experience. J Clin Oncol. 2010;28:318-23.

2. Dudanakar MPKMKM, Agnihotri NS. Intraoral fibrosarcomea of tongue: An unusual case report with review. Int $\mathrm{J}$ of Develop Res. 2015;5:3836-9.

3. Wadhwan VCM, Gawande M. Fibrosarcoma of the oral cavity. Indian J Dent Res. 2010;21:295-8.

4. Sheng WQ, Hisaoka M, Okamoto S. A clinicopathologic study of 10 cases and molecular detection of the ETV6-NTRK3 fusion transcripts using paraffin-embedded tissues. Am J Clin Pathol. 2001;115:348-55.

5. Parida L, Fernandez-Pineda I, Uffman JK, Davidoff AM, Krasin MJ, Pappo A, et al. Clinical management of infantile fibrosarcoma: a retrospective single-institution review. Pediatr Surg int. 2013;29:703-8.

6. Gupta A, Maddalozzo J, Htin TW, Shah A, Chou PM. Spindle cell rhabdomyosarcoma of the tongue in an infant: a case report with emphasis on differential diagnosis of childhood spindle cell lesions. Pathol Res Practice. 2004;200:537-43.

7. Laco J, Simakova E, Slezák R, Tucek L, Mottl R, Spacek J, et al. Low grade myofibroblastic sarcoma of tongue: a case report. Cesk Patol. 2006;42:150-3.

8. Wiswell Te, Sakas El, Stephenson Sr, Lesica Jj, Reddoch Sr. Infantile myofibromatosis. Pediatrics. 1985;76:981-4.

AFFILIATION:

Department of ENT, Head and Neck Surgery Medical Teaching Institute (MTI), Lady Reading Hospital (LRH), Peshawar-Pakistan

\section{CORRESPONDENCE:*}

